“ (C) 2016 IEEE. Personal use of this material is permitted. Permission from IEEE must be obtained for all other uses, in any current or future media, including

reprinting/republishing this material for advertising or promotional purposes, creating new collective works, for resale or redistribution to servers or lists, or reuse of any copyrighted component of this work in other works." 


\title{
Flexible Uniplanar Electrically Small Directive Antenna Empowered by a Modified CPW-feed
}

\author{
Ming-Chun Tang, Member, IEEE, Boya Zhou, and Richard W. Ziolkowski, Fellow, IEEE
}

\begin{abstract}
A flexible printed near-field resonant parasitic (NFRP) GPS L1 antenna that is a compact, efficient, directive radiator is demonstrated. The simple uniplanar design incorporates a capacitively loaded loop (CLL) resonator, which acts as the NFRP element, and a coplanar waveguide (CPW)-fed semi-loop antenna. A set of slots are introduced into the CPW feedline ground strips. The resulting meanderline-shaped CPW ground strips act as a driven dipole element that is capacitively coupled to the NFRP element in such a manner that when they are properly tuned, nearly complete matching to the $50 \Omega$ source is achieved with no matching circuit, and the pair acts as a two-element end-fire array. Parameter studies are reported to illustrate the nuances of the design and its operating mechanisms. The experimental results are in good agreement with their simulated values. The end-fire realized gain is $3.57 \mathrm{dBi}$ with a $13.44 \mathrm{~dB}$ front-to-back-ratio (FTBR) at its resonance frequency: 1.574 GHz (GPS L1), where the electrical size $k a=0.97$. The flexibility of the proposed antenna is demonstrated both numerically and experimentally by mounting it on several cylindrical structures whose curvatures vary over a large range and by confirming that there is little impact on its operational frequency, impedance matching, bandwidth and radiation characteristics.
\end{abstract}

Index Terms -Directivity, electrically small antennas, end-fire array, flexible antennas, printed antennas, uniplanar antennas

\section{INTRODUCTION}

A $\mathrm{s}$ the desire for yet smaller mobile platforms has increased, the need for printed electrically small antennas (ESAs) has grown intense. Generally, omni-directional patterns are desired and they arise naturally from electric or magnetic dipole radiators $[1,2]$, which occur when the size of the radiating structure is much smaller than the source wavelength. Nonetheless, there are numerous applications, including biomedical monitoring and on-body systems; point-to-point communications and wireless power transfer; and radio frequency identification devices (RFIDs), for which it is desirable to achieve higher directivity and/or a large

Manuscript received on Jun. 23, 2015; revised on Aug. 06, 2015

This work was supported in part by the National Natural Science Foundation of China contract number 61471072, and in part by the Fundamental Research Funds for the Central Universities contract number 106112015CDJZR165510.

M. -C. Tang and B. Zhou are with the College of Communication Engineering, Chongqing University, Chongqing, 400044, China (E-mail: tangmingchunuestc@126.com);

R. W. Ziolkowski is with the Department of Electrical and Computer Engineering, University of Arizona, Tucson, AZ 85721 USA (E-mail: ziolkowski@ece.arizona.edu). front-to-back ratio, i.e., to have the radiated power emitted primarily into one hemisphere.

There have been many approaches reported that obtain higher directivity with ESAs. One was enabled by the development of high impedance ground planes $[3,4]$ and bulk artificial magnetic conductors [5], i.e., meta-structures and metamaterials acting as in-phase reflectors. A second is to use two ESAs in an end-fire array configuration [6-10]. A third is to introduce a meanderline slot-based near-field resonant parasitic element (NFRP) tuned specifically for directivity enhancement $[11,12]$. A fourth is to consider an orthogonal combination of electric and magnetic dipole radiators to achieve a Huygens source $[13,14]$.

In this letter a coplanar waveguide (CPW)-fed, flexible printed uniplanar NFRP ESA is reported that achieves high directivity away from the feed at the GPS L1 frequency. The $\mathrm{CPW}$-feedline drives a semi-loop antenna. Following the metamaterial-inspired NFRP ESA paradigm [15], a capacitively loaded loop (CLL) element is introduced near to the driven element. Then, in analogy with [16] and [11, 12], the CPW ground strips are modified into a meanderline form to facilitate the enhanced directivity. It will be demonstrated that the modified CPW ground strips act as a dipole element whose currents are driven by the semi-loop out-of-phase with the CLL NFRP element yielding a two element end-fire array. An experimental prototype was fabricated and tested. The simulated and measured performance characteristics will be shown to be in good agreement. The prototype was then mounted on several cylindrical structures and tested. It will be shown that the operating characteristics of this conformal antenna vary little even in the presence of significant bending. The flexible nature of the antenna in a uniplanar format is very practical for engineering applications. In particular, since it employs only one thin metallic layer, there is no need for precise alignment among layers in the manufacturing process, which, in practice, leads to smaller processing/fabrication errors. It also avoids inconvenient hardening of cloth substrates in textile variants. Furthermore, it could be easily packaged directly with microwave monolithic integrated circuits (MMICs) or with complementary metal oxide semiconductor (CMOS) RF circuitry without impacting their corresponding strict process design rules.

Note that all of the numerical simulations reported in this article were performed using the frequency domain ANSYS/ANSOFT high frequency structure simulator (HFSS), 
version 13.0. All simulations were performed with known, experimentally obtained material parameters.

\section{Modified-CPW GRound STRIP-BASED Design}

The evolution of the design of the reported antenna is shown in Fig. 1. The illustrated antennas are constructed from 0.018-mm-thick copper, $0.127 \mathrm{~mm}$ thick Rogers 5880 board material $\left(\varepsilon_{r}=2.2, \mu_{r}=1.0\right.$, and $\left.\tan \delta=0.0009\right)$. The copper was modeled in all cases with its known parameters: $\varepsilon_{r}=1.0, \mu_{r}=$ 0.999991 and bulk conductivity $\sigma=5.8 \times 10^{7} \mathrm{~S} / \mathrm{m}$. The detailed dimensions are given in Table I. The first configuration, shown in Fig. 1(a), is simply a typical CPW-fed monopole antenna. The CPW line feeds a semi-loop antenna printed on Rogers Duroid $^{\mathrm{TM}} 5880$ board material. The CPW line is directly fed via a $50 \Omega$ coaxial cable. The center conductor is connected to the driven strip and the two ground strips are connected to the outer metallic part of the coaxial cable.

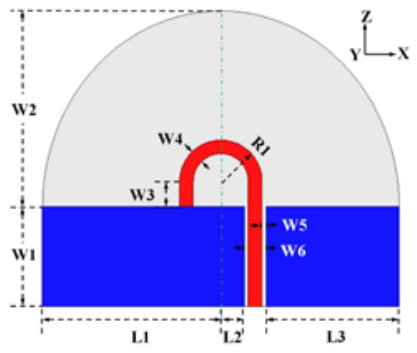

(a)

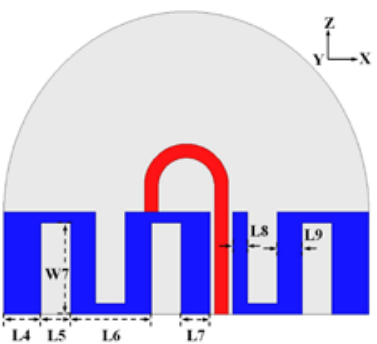

(b)

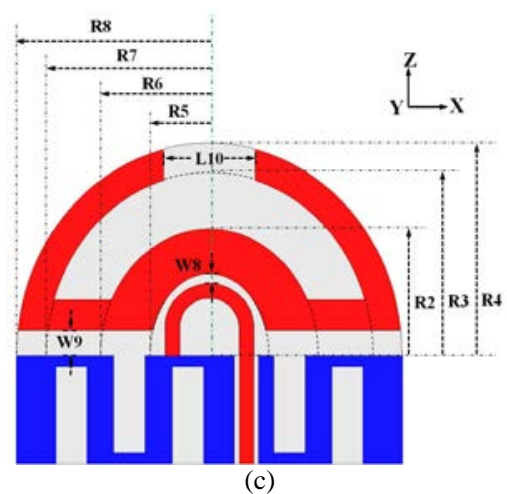

Fig. 1 Evolution of the uniplanar ESA. (a) Typical CPW-fed monopole antenna; and CPW-fed semi-loop antenna (b) with shaped meanderline ground strips, and then (c) augmented with the CLL NFRP element.

TABLE1. The Geometrical Parameters of Three UniPlanar ESAs (All DiMENSIONS ARE IN MiLliMETERS, AND R5, R6, R7 AND R8 REPRESENT THE Distance Between Center Point AND the TANGent of Ellipse)

(a) Typical CPW-Fed Monopole ANTENNA

\begin{tabular}{|c|c|c|c|c|}
\hline$L 1=26$ & $L 2=3.85$ & $L 3=19.85$ & $W 1=14.5$ & $W 2=28.5$ \\
\hline$W 3=3.7$ & $W 4=2$ & $W 5=0.15$ & $W 6=2.3$ & $R 1=6$ \\
\hline
\end{tabular}

(b) CPW-FED ESA with MEANDER-Line SHAPED GROUND STRIPS

\begin{tabular}{|l|l|l|c|c|}
\hline$L 4=5.3$ & $L 5=4.2$ & $L 6=11.5$ & $L 7=4.65$ & $L 8=2.55$ \\
\hline$L 9=3.6$ & $W 7=13$ & \multicolumn{4}{|c|}{ Null } \\
\hline
\end{tabular}

(c) UNIPLANAR CPW-FED DiRECTIVE ESA

\begin{tabular}{|c|c|c|c|c|}
\hline$L 10=12.3$ & $W 8=1.3$ & $W 9=3.4$ & $R 2=17$ & $R 3=24.5$ \\
\hline$R 4=28.5$ & $R 5=8$ & $R 6=14.7$ & $R 7=22$ & $R 8=26$ \\
\hline
\end{tabular}

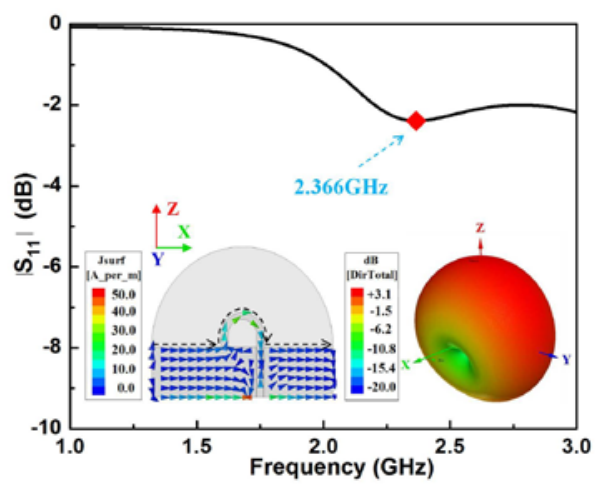

(a)

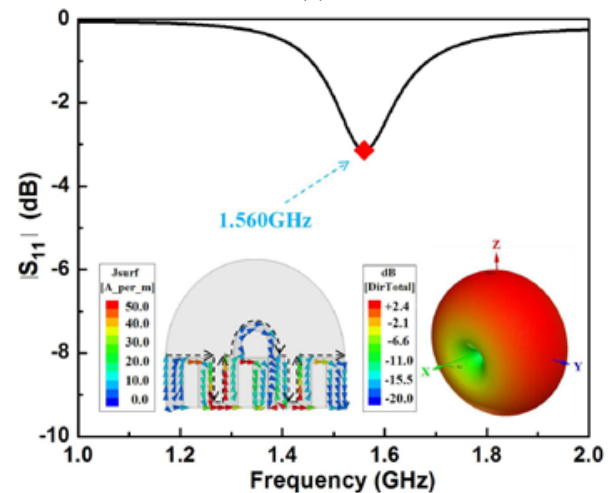

(b)

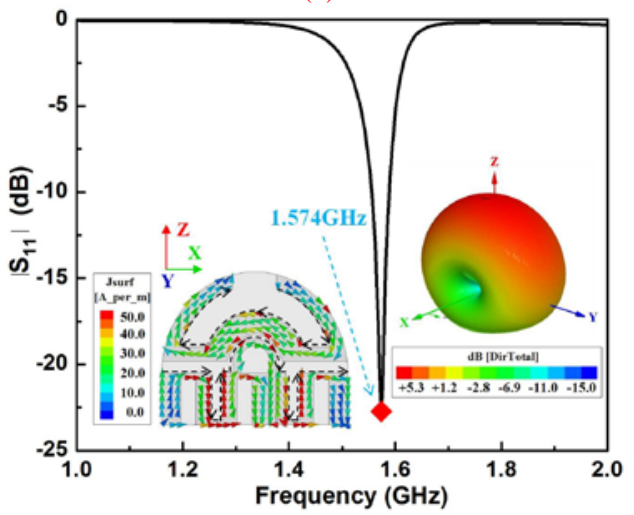

(c)

Fig. 2 Simulated response characteristics: $\left|\mathrm{S}_{11}\right|$ values in $\mathrm{dB}$ versus the source frequency, the surface current density vector and amplitude distributions, and the 3-D directivity patterns at the resonance frequency for the three uniplanar antennas. (a) Typical CPW-fed monopole antenna; and CPW-fed semi-loop antenna (b) with shaped meanderline ground strips, and then (c) augmented with the CLL NFRP element.

The corresponding response characteristics: $\left|\mathrm{S}_{11}\right|$ values $(\mathrm{dB})$ as a function of the source frequency; the surface current density distribution and vectors; and the 3D directivity patterns are shown in Fig. 2(a). Although not shown, we note that in the HFSS model, a short coaxial cable with length $5.0 \mathrm{~mm}$ is present to increase the simulation accuracy. It is clear that matching was not achieved; $\left|\mathrm{S}_{11}\right|_{\min }=-2.40 \mathrm{~dB}$ at $2.366 \mathrm{GHz}$. With $a$ being the radius of the smallest sphere that completely encloses the antenna at the resonance frequency, $f_{0}$, and $k=$ $2 \pi / \lambda_{0}=2 \pi f_{0} / c$ is the free space wave number, its electrical size at resonance is $k a=1.454$. At $f_{0}$ the currents on the combined semi-loop and ground strips radiate as an electric dipole oriented along the $\mathrm{x}$-axis. In fact, the current path length, as indicated by the arrowed dashed line, is $66.1 \mathrm{~mm}=0.52 \lambda_{0}$. 
Five rectangular slots with the same size (L5×W7) were etched from the ground strips, while maintaining the CPW-feed line, to achieve the semi-loop antenna augmented with the meanderline ground strips shown in Fig. 1(b). The corresponding response characteristics are shown in Fig. 2(b). A resonance is now present at the lower frequency: $1.560 \mathrm{GHz}$, where $\left|\mathrm{S}_{11}\right|_{\min }=-3.14 \mathrm{~dB}$, enabled by the inductance associated with the meanderline. Consequently, the antenna is now electrically small with $k a=0.96$. The antenna still radiates as an electric dipole along the $\mathrm{x}$-axis. The directivity pattern remains basically axisymmetric about the x-axis. The meanderline provides a slightly longer pathway: $117.6 \mathrm{~mm}\left(\sim 0.61 \lambda_{0}\right)$ for the current but within the same total physical size. This self-resonant current distribution behavior is similar to that in Fig. 2(a). It also produces dipole radiation characteristics with the maximum directivity value being $2.4 \mathrm{~dB}$.

Finally, a CLL NFRP element that conforms to the semi-loop antenna to maximize their interaction was introduced as shown in Fig. 1(c). We used HFSS to establish the trends and tradeoffs when the dimensions were varied through parameter studies. The predicted response characteristics for the optimized design are shown in Fig. 2(c). Nearly complete impedance matching to the $50 \Omega$ source was achieved, i.e., $\left|S_{11}\right|_{\min }=-22.72 \mathrm{~dB}$, at the resonance frequency, the GPS L1 value: $1.574 \mathrm{GHz}$. We emphasize that this is achieved without resort to any balun, transition, or complicated feed network. The -10dB impedance bandwidth was $32.5 \mathrm{MHz}$. Furthermore, the electrical size of the antenna was maintained at $k a=0.967$. It produces strong end-fire directivity, including a $5.26 \mathrm{~dB}$ peak directivity strictly along the z-axis, a $14.58 \mathrm{~dB}$ FTBR, and a $96.07 \%$ radiation efficiency. Thus, its peak realized gain value in the end-fire direction is $5.16 \mathrm{dBi}$. By inspecting the surface current distribution behavior, one observes that the current induced on the CLL element is nearly $180^{\circ}$ out of phase with that on the meanderline, but has a comparable magnitude. Thus, the basic requirement for a two-element end-fire array design was met $[6,7]$. We note that the total current pathway on the CLL element is $110.86 \mathrm{~mm}\left(0.58 \lambda_{0}\right)$, which is shorter than that on the meanderline ground strips: $117.6 \mathrm{~mm}\left(0.62 \lambda_{0}\right)$. Moreover, because of its symmetrical excitation and its resonant length, the current density on the CLL element radiates as an electric dipole rather than as a magnetic one. Therefore, the CLL element radiates as an electric dipole and acts as a NFRP director for the resulting two-element electric dipole end-fire (quasi-Yagi) array. This was confirmed further by designing a version in which the CLL NFRP element is longer than the meanderline and, consequently, acts as a reflector element. The maximum directivity was then found to be along the $-\mathrm{z}$-axis.

\section{EXPERIMENTAL VALIDATION}

A prototype of the optimized antenna was fabricated and measured in an anechoic chamber at the University of Electronic Science and Technology of China (UESTC) having a SATIMO passive measurement system. The $\left|\mathrm{S}_{11}\right|$ values were obtained for different source frequencies using an Agilent E8361A PNA Vector Network Analyzer (VNA). The measured results in Fig. 3 show that the antenna had a $1.5835 \mathrm{GHz}$ resonance frequency with a nearly complete impedance match to the $50 \Omega$ source $\left(\left|\mathrm{S}_{11}\right|_{\min }=-19.27 \mathrm{~dB}\right)$ and a $-10 \mathrm{~dB}$ impedance bandwidth equal to $30.2 \mathrm{MHz}$, while its electrical size was $k a=$ 0.973 . The simulated and measured far-field realized gain patterns shown in Fig. 4 are also in good agreement.

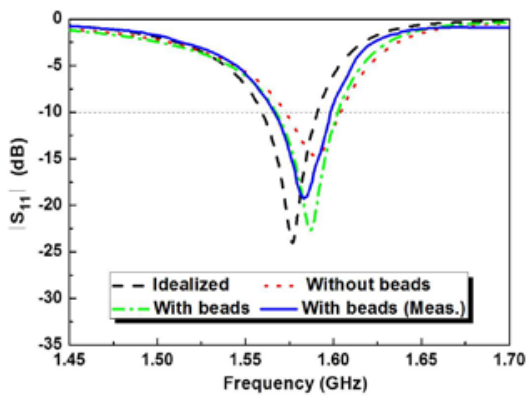

Fig. 3 Comparison of the simulated and measured $\left|S_{11}\right|$ values for the fabricated prototype under different measurement conditions.

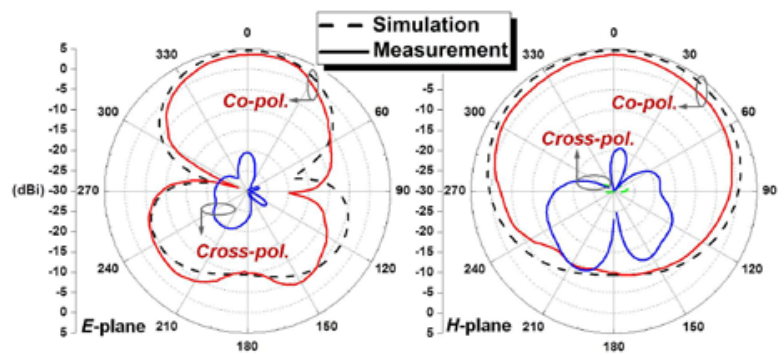

(a)

(b)

Fig. 4 Comparison of the simulated and measured 2D realized gain patterns of the prototype antenna at its resonance frequency: $1.574 \mathrm{GHz}$ and $1.5835 \mathrm{GHz}$, respectively, in the (a) $E$ - (ZOX) and (b) $H$ - (ZOY) planes.

We note that because of its size and configuration, the fields radiated by the antenna coupled significantly to the long coax measurement cable, which was connected to the antenna under test (AUT). The negative effect this coupling had is illustrated in Fig. 3. In order to suppress the surface current on the outer conductor of the coax measurement cable, a periodic arrangement of ferrite beads provided by the Southwest China Institute of Applied Magnetic Technology were placed on it. This approach was successful in mitigating the effects of those currents, as shown in Fig. 3. The maximum end-fire realized gain and FTBR shown in Fig. 4 are $3.57 \mathrm{dBi}$ and $13.44 \mathrm{~dB}$, respectively. These values are lower than the simulated ones without the beads, but are in agreement with those with them. The antenna is seen to produce a far field with a high linear polarization purity. The measured (simulated) crosspolarization level was lower by $-24.11 \mathrm{~dB}$ (smaller than $-30 \mathrm{~dB}$ in simulation and, as a result, these results are not present in the plot) in the end-fire direction. Additional reasons leading to the differences between the measured and simulated values: resonance frequency red-shift $(\sim 3.5 \mathrm{MHz})$, peak gain reduction ( $1.04 \mathrm{~dB}$ ), and the tilt of the two dips in the E-plane arise from the lack of mechanical stability under measurement of this extremely thin antenna, unavoidable errors in the fabrication processes, and some minor errors in the measurement process. On the whole, the measurement results are in agreement with their simulated values.

\section{FLEXIBILITY STUDY}

The flexibility of the antenna was investigated both numerically and experimentally by mounting it on Styrofoam 
cylinders having different radii of curvature: 20 to $120 \mathrm{~mm}$ in $20 \mathrm{~mm}$ increments. The antenna, bent vertically and horizontally, and mounted conformally on the corresponding $20 \mathrm{~mm}$ cylinder, is shown, respectively, in Figs. 5(a) and 5(b). The simulated and measured results for each of the vertical cases are presented in Tables II. These results include the resonance frequency, $\left|S_{11}\right|_{\text {min }}$ values, $-10 \mathrm{~dB}$ impedance bandwidth (BW), maximum end-fire realized gain values (idealized versions in simulation and with beads in experiment), FTBR, and radiation efficiency values (RE).

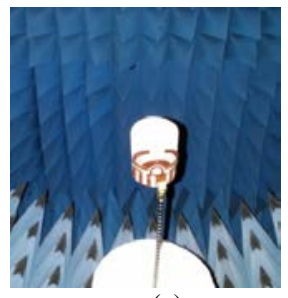

(a)

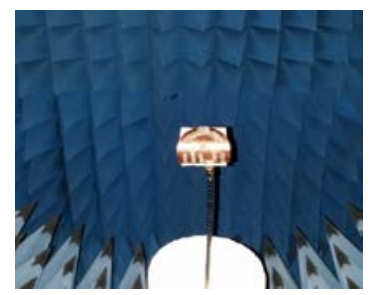

(b)
Fig. 5 The prototype flexible antenna mounted on a $20 \mathrm{~mm}$ radius foam cylinder in the anechoic chamber. (a) Vertical bend, and (b) horizontal bend.

TABLE II. RADIATION CHARACTERISTICS OF THE PROTOTYPE ANTENNA WITH DiFFERENT AMOUNTS OF VERTICAL BENDING

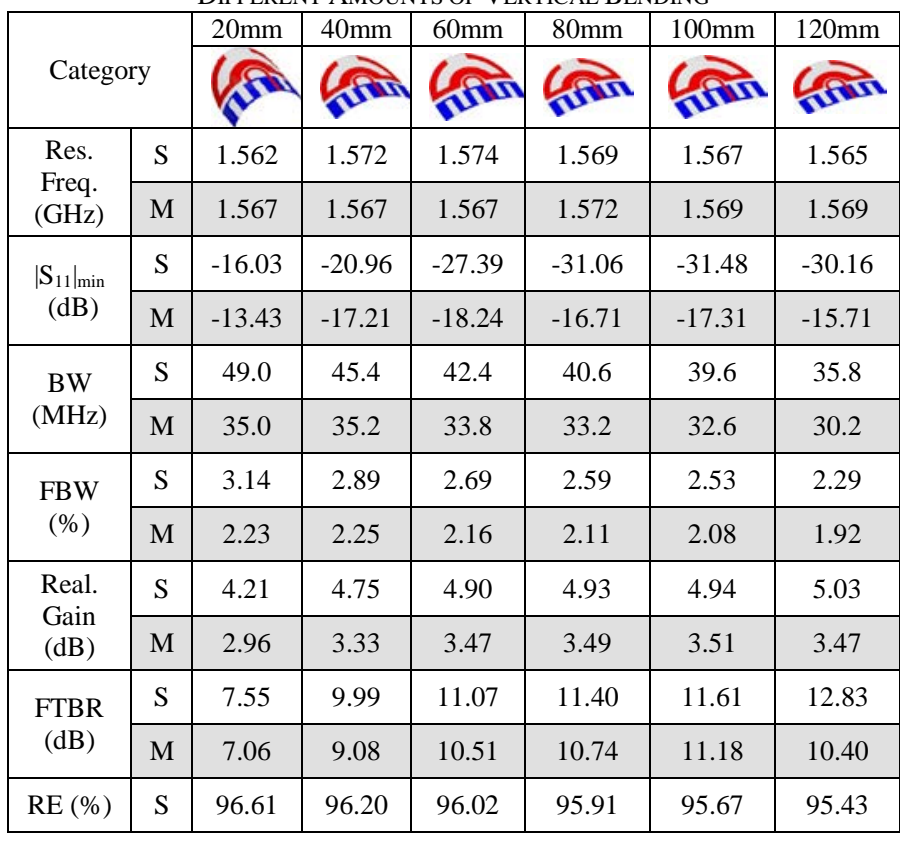

From Table II, it is clear that the effects of the bending in vertical direction on the performance characteristics are reasonable from a practical perspective. The resonance frequency and the level of impedance matching, as well as the BW, FBW, RG, FTBR, and RE values support this observation for all of the tested conformal configurations. We note that an increase of the foam radius is equivalent to a reduction of the curvature of the antenna and, consequently, all of the variations in these values consistently change to approach the corresponding planar values given previously. Conversely, the smaller the radius of curvature is, the larger are the variations. While basically the same performance characteristics were obtained numerically and experimentally for the horizontal bending configurations, we note that the vertical bending cases do exhibit smaller variations from the planar values than the associated horizontal bending ones.

\section{CONCLUSIONS}

A simple, uniplanar, flexible, printed, electrically small, GPS L1 antenna with efficient end-fire, high directivity radiation characteristics was reported. The ground strips of its CPW-fed semi-loop antenna were modified into a meanderline to significantly reduce the resonance frequency and, hence, its electrical size. The addition of a CLL NFRP element produced an end-fire (away from the source) behavior with realized gain and FTBR values as high as $3.57 \mathrm{dBi}$ and $13.44 \mathrm{~dB}$, respectively, despite its electrically small size: $k a<1$ and nearly complete impedance matching without any complicated feed network. It radiation characteristics were treated numerically and confirmed experimentally. Its flexibility was tested extensively by mounting it conformally on a large set of cylindrical structures. The numerical and experimental results demonstrate that the antenna exhibits a very low susceptibility to changes in its radiation performance characteristics under different bending and curvature conditions.

\section{REFERENCES}

[1] R. F. Harrington, "On the gain and beamwidth of directional antennas," IEEE Trans. Antennas Propag., vol. 6, no. 3, pp. 219-225, Jul. 1958.

[2] R. F. Harrington, "Effect of antenna size on gain, bandwidth and efficiency,” J. Res. Nat. Bur. Stand, vol. 64-D, pp. 1-12, Jan./ Feb. 1960.

[3] F. Yang and Y. Rahmat-Samii, Electromagnetic Band Gap Structures in Antenna Engineering. Cambridge: UK: Cambridge University Press, 2009.

[4] P. Jin, and R. W. Ziolkowski, "High-directivity, electrically small, low-profile near-field resonant parasitic antennas,” IEEE Antennas Wirel. Propag. Lett., vol. 11, pp. 305-309, 2012.

[5] A. Erentok, P. Luljak, R. W. Ziolkowski "Antenna performance near a volumetric metamaterial realization of an artificial magnetic conductor," IEEE Trans. Antennas Propag., vol. 53, no. 1, pp. 160-172, Jan. 2005.

[6] E. E. Altshuler, T. H. O’Donnell, A. D. Yaghjian, and S. R. Best, "A monopole superdirective array,” IEEE Trans. Antennas Propag., vol. 53, no.8, pp. 2653-2661, Aug. 2005.

[7] A. D. Yaghjian, T. H. O'Donnell, E. E. Altshuler, and S. R. Best, "Electrically small supergain end-fire arrays," Radio Sci., vol.43, no. RS3002, pp. 1-13, 2008.

[8] S. Lim, and H. Ling, "Design of a closely spaced, folded Yagi antenna," IEEE Antennas Wirel. Propag. Lett., vol. 5, pp. 302-305, 2006.

[9] A. Noguchi, and H. Arai, "3-element super-directive end-fire array with decoupling network," Proceedings of ISAP 2014, Kaohsiung, Taiwan, Dec. 2-5, 2014.

[10] M.-C. Tang, R. W. Ziolkowski, S. Xiao, and M. Li, "A high-directivity, wideband, efficient, electrically small antenna system,” IEEE Trans. Antennas Propag., vol. 62, no. 12, pp. 6541 - 6547, Dec. 2014.

[11] M.-C. Tang, and R. W. Ziolkowski, "Efficient, high directivity, large front-to-back-ratio, electrically small, near-field-resonant-parasitic antenna," IEEE Access, vol. 1, no. 1, pp. 16 - 28, May 2013.

[12] R. W. Ziolkowski, M.-C. Tang and N. Zhu, "An efficient, broad bandwidth, high directivity, electrically small antenna," Microw. Opt. Technol. Lett., vol. 55, no. 6, pp. 1430-1434, June 2013.

[13] P. Jin, and R. W. Ziolkowski, "Metamaterial-inspired, electrically small Huygens sources," IEEE Antennas Wirel. Propag. Lett., vol. 9, pp. 501-505, 2010.

[14] T. Niemi, P. Alitalo, A. O. Karilainen, and S.A. Tretyakov, "Electrically small Huygens source antenna for linear polarization," IET Microw. Antennas Propag., Vol. 6, Iss. 7, pp. 735-739, 2012.

[15] R. W. Ziolkowski, P. Jin, and C.-C. Lin, "Metamaterial-inspired engineering of antennas,” Proc. IEEE, vol.99, no.10,pp.1720-1731,Oct. 2011.

[16] A. T. Mobashsher and R. W. Aldhaheri, "An improved uniplanar front-directional antenna for dual-band RFID readers," IEEE Antennas Wirel. Propag. Lett., vol. 11, pp. 1438-1441, 2012. 